\title{
RELATIONS BETWEEN STIEFEL-WHITNEY CLASSES OF MANIFOLDS
}

BY EDGAR H. BROWN, JR. AND FRANKLIN P. PETERSON ${ }^{1}$

Communicated by W. S. Massey, November 1, 1962

1. Introduction. Let $M$ be a $C^{\infty}$-manifold and let $\tau_{M}: M \rightarrow B O$ be the classifying map of its stable tangent bundle. Recall that $H^{*}\left(B O ; Z_{2}\right)$ is a polynomial algebra on the $W$ hitney classes $W_{1}, W_{2}, \cdots, W_{n}, \cdots$. Define the ideal $I_{n} \subset H^{*}\left(B O ; Z_{2}\right)$ of relations between Stiefel-Whitney classes of manifolds of dimension $n$ as follows:

$$
I_{n}=\bigcap \operatorname{Ker} \tau_{M}^{*}
$$

where $M$ ranges over all $n$-dimensional, compact, connected, $C^{\infty}$ manifolds without boundary.

Let $I_{n}^{k}$ denote the elements of $I_{n}$ of dimension $k . \mathrm{E}$. H. Brown [2] and R. Stong have shown that $I_{n}^{k}=0$ if $k \leqq n / 2$. A. Dold [3] has calculated $I_{n}^{n}$. In this paper we compute $I_{n}^{k}$ for all $n$ and $k$ and furthermore show, in a sense to be made precise in $\$ 3$, that all of these relations are algebraic in character. In $\$ 2$ we give the preliminary definitions necessary for the statement of our results and in $\$ 3$ we give these results.

\section{Right action of the Steenrod algebra. Let}

$$
H=\sum_{k=0}^{n} H^{k}
$$

be a graded commutative algebra with unit over $Z_{2}$ which is of finite type. Assume $A$, the mod 2 Steenrod algebra, acts on the left of $H$ as a Hopf algebra (see [4]). This means that the Cartan formula holds, $S q^{i}(h)=h^{2}$ if $\operatorname{dim}(h)=i$ and $S q^{i}(h)=0$ if $\operatorname{dim}(h)<i$. Furthermore assume $H$ satisfies Poincare duality. That is, $H^{n} \approx Z_{2}$ and $h \in H^{i}$ is zero if and only if $h h^{\prime}=0$ for all $h^{\prime} \in H^{n-i}$. Such an algebra will be called a Poincaré algebra. Following Adams [1], we define a right operation of $A$ on $H$ by the condition:

$$
h a \cdot h^{\prime}=h \cdot a h^{\prime}
$$

for all $h^{\prime} \in H^{n-i-i}$ where $h \in H^{i}$ and $a \in A_{j}$. Define

1 The first named author is an NSF Senior Postdoctoral Fellow and was partially supported by an NSF research grant, and the second named author is an Alfred P. Sloan Fellow and was partially supported by the U. S. Army Research Office. 


$$
\begin{aligned}
v_{i} & =(1) S q^{i}, \\
W_{i} & =\sum_{j=0}^{i} S q^{i}\left(v_{i-j}\right), \\
\bar{W}_{i} & =\sum_{j=0}^{i-1} \bar{W}_{j} W_{i-j}, \quad \bar{W}_{0}=1 .
\end{aligned}
$$

It is not difficult to prove the following theorem.

Theorem 2.1. $(h) \chi\left(S q^{i}\right)=\sum_{j=0}^{i} \bar{W}_{j} \cdot S q^{i-i}(h)$ where $\chi$ is the canonical anti-automorphism of $A$.

Suppose $U$ is a graded commutative algebra with unit over $Z_{2}$ on which $A$ acts on the left as a Hopf algebra. Let $u_{i} \in U^{i}, i=0,1,2, \cdots$, where $u_{0}=1$. Following Theorem 2.1 we may attempt to define a right action of $A$ on $U$ by the formula:

$$
\text { (u) } \chi\left(S q^{i}\right)=\sum_{j=0}^{i} u_{j} \cdot S q^{i-j}(u) .
$$

In general this formula will not be consistent with the Adem relations.

THEOREM 2.3. The formula (2.2) makes $U$ into a right module over $A$ if and only if the $u_{i}$ satisfy the $W u$ formulae, i.e.

$$
S q^{r}\left(u_{i}\right)=\sum_{t=0}^{r}\left(\begin{array}{c}
i-r+t-1 \\
t
\end{array}\right) u_{r-t} u_{i+t} .
$$

Corollary 2.4. If $\bar{W}_{i}=u_{i}$, (2.2) makes $H^{*}\left(B O ; Z_{2}\right)$ into a right module over $A$.

COROLlaRy 2.5. If $A$ acts on the right of $U$ according to the formula (2.2), then there is a unique algebra homomorphism $\tau_{U}: H^{*}\left(B O ; Z_{2}\right) \rightarrow U$ which is equivariant with respect to the right and left actions of $A$.

3. Relations between Stiefel-Whitney classes. Let $S \subset H^{*}\left(B O ; Z_{2}\right)$. Define $I_{n}(S$, geom $)=\bigcap \operatorname{Ker} \tau_{M}^{*}$ where $M$ runs over all $n$-dimensional, compact, $C^{\infty}$-manifolds without boundary such that $\tau_{M}^{*}(S)=0$. Note $I_{n}(\phi$, geom $)=I_{n}$. Similarly, define

$$
I_{n}(S, \operatorname{alg})=\bigcap \operatorname{Ker} \tau_{H}
$$

where $H$ ranges over all $n$-dimensional Poincaré algebras such that $\tau_{H}(S)=0$. Clearly $I_{n}(S$, alg $) \subset I_{n}(S$, geom $)$.

Let $F_{n} \subset H^{*}\left(B O ; Z_{2}\right)$ be the $Z_{2}$ module generated by $H^{i}\left(B O ; Z_{2}\right) S q^{i}$ for all $i$ and $j$ such that $2 i>n-j$. Note that $F_{n} \subset I_{n}(\phi$, alg $)$, for if $x \in H^{i}\left(B O ; Z_{2}\right)$ and $2 i>n-j, \tau_{H}\left((x) S q^{i}\right) \cdot h^{\prime}=\tau_{H}(x) \cdot S q^{i}\left(h^{\prime}\right)=0$ for all $h^{\prime} \in H^{n-i-j}$. Our main theorem is the following: 
THEOREM 3.1. $I_{n}=F_{n}$.

Corollary 3.2. $I_{n}=I_{n}(\phi, \mathrm{alg})$.

COROLLARY 3.3 .

(a) $I_{n}^{k}=0$ if $k \leqq n / 2$.

(b) $I_{n}^{[n / 2]+1}$ is the $Z_{2}$ module generated by (1) $S q^{[n / 2]+1}$.

(c) $I_{n}^{[n / 2]+2}$ is the $Z_{2}$ module generated by (1) $\mathrm{Sq}^{[n / 2]+2}$, $W_{1}\left((1) S q^{[n / 2]+1}\right)$ and $S q^{1}\left((1) S q^{[n / 2]+1}\right)$.

(d) $I_{n}^{n}$ is the $Z_{2}$ module generated by $\left(S q^{i}+(1) S q^{i} \cdot\right) H^{n-i}\left(B O ; Z_{2}\right)$ for $i=1,2, \cdots, n$.

REMARK. 3.3(a) is the theorem of Brown and Stong and 3.3(d) is the theorem of Dold [3].

REMARK. $I_{n}$ contains the smallest ideal containing (1) $S q^{i}, i>n / 2$, which is closed under the right and left actions of $A$ but this ideal does not equal $I_{n}$.

$I_{n}(S$, alg) may be characterized in the following fashion. Let $J(S)_{q} \subset H^{*}\left(B O ; Z_{2}\right) \otimes H^{*}\left(K\left(Z_{2}, q\right), Z_{2}\right)$ be the ideal generated by $S \otimes 1$. Let $L_{n, q} \subset H^{*}\left(B O ; Z_{2}\right) \otimes H^{*}\left(K\left(Z_{2}, q\right), Z_{2}\right)$ be the $Z_{2}$ module generated by all elements of the form:

$$
\sum_{=0}^{i} S q^{j}(u) \otimes S q^{i-i}(x)+(1) S q^{i} \cdot u \otimes x
$$

where $i+\operatorname{dim} u+\operatorname{dim} x=n$. Let $\iota^{q} \in H^{q}\left(K\left(Z_{2}, q\right), Z_{2}\right)$ be the canonical generator.

TheOREM 3.4. $u \in I_{n}^{k}\left(S\right.$, alg) if and only if $u \otimes \iota^{n-k} \in L_{n, n-k}+J(S)_{n-k}$.

REMARK. It is not true that $I_{n}\left(\left\{W_{1}\right\}\right.$, geom) is the ideal generated by $I_{n}$ and $W_{1}$.

REMARK. It is not true that for all $S, I_{n}(S$, geom $)=I_{n}(S$, alg $)$.

\section{BIBLIOGRAPHY}

1. J.F. Adams, On formulae of Thom and Wu, Proc. London Math. Soc. 11 (1961), 741-752.

2. E. H. Brown, Nonexistence of low dimension relations between Stiefel-Whitney classes, Trans. Amer. Math. Soc. 104 (1962), 374-382.

3. A. Dold, Vollständigkeit der Wuschen Relation zwischen den Stiefel-Whitneyschen Zahlen differenzierbarer Mannigfaltigkeiten, Math. Z. 65 (1956), 200-206.

4. N. E. Steenrod, The cohomology algebra of a space, Enseignement Math. 7 (1961), 153-178.

BRANDEIS UNIVERSITY,

The Institute for Advanced Study and

Massachusetts Institute of Technology 